Research Paper

\title{
FOXC1 Contributes To Microvascular Invasion In Primary Hepatocellular Carcinoma Via Regulating Epithelial-Mesenchymal Transition
}

\author{
Zhi-Yuan Xu ${ }^{1,2^{*}}$, Song-Ming Ding ${ }^{1,2^{*}}$, Lin Zhou ${ }^{1,2^{*}}$, Hai-Yang Xie ${ }^{1,2}$, Kang-Jie Chen ${ }^{1,2}$, Wu Zhang ${ }^{1,2}$, Chun-Yan \\ Xing ${ }^{1,2}$, Hai-Jun Guo ${ }^{1,2}$, and Shu-Sen Zheng ${ }^{1,2 \varpi}$ \\ 1. Key Laboratory of Combined Multi-organ Transplantation, Ministry of Public Health; Key Laboratory of Organ Trans- \\ plantation, Zhejiang Province; Hangzhou, Zhejiang, China; \\ 2. Division of Hepatobiliary and Pancreatic Surgery, Department of Surgery, First Affiliated Hospital, Zhejiang University \\ School of Medicine; Hangzhou, Zhejiang, China. \\ * These authors contributed equally to this work.
}

\begin{abstract}
$\bowtie$ Corresponding author: Shu-sen Zheng, PhD, MD, FACS. E-mail: shusenzheng@zju.edu.cn Tel: 86-571-87236570 Fax: 86-571-87236628
( ) Ivyspring International Publisher. This is an open-access article distributed under the terms of the Creative Commons License (http://creativecommons.org/ licenses/by-nc-nd/3.0/). Reproduction is permitted for personal, noncommercial use, provided that the article is in whole, unmodified, and properly cited.
\end{abstract}

Received: 2012.06.21; Accepted: 2012.08.28; Published: 2012.09.08

\begin{abstract}
The existence of microvascular invasion (MVI) formation is one of the most important risk factors predicting poor outcome in hepatocellular carcinoma (HCC) and its mechanism remains largely unknown. Epithelial-Mesenchymal Transition (EMT) has been suggested to be involved in many steps of the invasion-metastasis cascade. To elucidate the possible contribution of EMT to MVI, we initially evaluated the expression of 8 EMT-related transcription factors (TFs) in HCC patients with or without MVI and found that $\mathrm{FOXCl}$ expression was significantly higher in patients with MVI than those without MVI $(P<0.05)$. Knockdown of FOXCI expression in HCC cells resulted in a partial conversion of their EMT progresses, mainly regulating the mesenchymal component. Ectopic expression of snail, twist or TGF- $\beta$ I could induce expression of FOXCI, but none of the expression of snail, twist, slug or TGF- $\beta$ was consistently down-regulated in response to $\mathrm{FOXCl}$ silencing, suggesting $\mathrm{FOXCl}$ might operate the downstream of other EMT regulators. In addition, knockdown of $\mathrm{FOXCl}$ expression led to cytoskeleton modification accompanied by decreased ability of cell proliferation, migration, and invasion. Meanwhile, some matrix metalloproteinases (MMPs) and VEGF-A were also simultaneously down-regulated. Together, our findings demonstrate that $\mathrm{FOXCl}$ is one of candidate predictive markers of $\mathrm{MVI}$, and that inhibition of $\mathrm{FOXCl}$ expression can partially reverse EMT program, offering a potential molecular therapeutic target for reducing tumor metastasis in HCC patients.
\end{abstract}

Key words: Hepatocellular carcinoma, Microvascular invasion, Epithelial-Mesenchymal Transition, FOXC1.

\section{Introduction}

Hepatocellular carcinoma (HCC) is the fifth most common cancer worldwide and the third leading cause of cancer death [1]. Currently, surgical treatment, including resection and transplantation, is an optional treatment strategy offering potential curative treatment and better long-term outcome in patients with HCC. Unfortunately, despite the expanded indications for surgical treatment with the technical advances, the rate of tumor recurrence and metastasis after curative resection remains high, which still limits patient survival [2]. Among the factors related to tumor recurrence, microvascular invasion (MVI) for- 
mation has been proved to be one of the most important risk clinicopathological factors to predict the metastatic potential of HCC [3]. The existence of MVI indicates that HCC has become systemic through invasion of peripheral blood vessel and subsequent spread [4]. However, microvascular invasion to minute peripheral areas, such as the third branch of the portal vein, is difficult to detect preoperatively with current conventional imaging modalities. In most cases, it can be only detected by pathological examination after surgery. No postoperative adjuvant treatment modalities including transcatheter arterial chemoembolization (TACE), can effectively improve the outcomes in this group of patients. This was consistent with numerous studies showing that early recurrence within 2 years is associated with MVI after curative liver resection and even orthotopic liver transplantation which totally eradicate the disease liver[5]. Hence, it is urgent to understand the underlying genetic basis for MVI and then seek measure to block it.

Increasingly, more attention is currently being given to changes in cellular architecture and polarity for insights into cancer behavior [6]. The epithelial-mesenchymal transition (EMT) is such process which allows polarized epithelial cells to acquire mesenchymal properties including fibroblastoid morphology, characteristic gene express changes, increasing potential for motility, and in the case of cancer, increased invasion, metastasis, resistance to chemotherapy [7]. The functional hallmark of carcinoma EMT is acquisition of ability to transmigration through extracellular matrix (ECM) as a single cell [8]. Notably, the carcinoma EMT has be regarded as a critical behavior for cancer cell to initial escape from primary site by endowing individual cell with enhanced invasive potential through barrier matrix and subsequent acquisition of intravasation, enabling cancer cells to enter the blood vessel[9]. Hence, we hypothesis that larger subpopulations of cells in primary HCC with MVI, compared with that without MVI, may have undergone an EMT for the purpose of generating MVI. Interesting, similar hypothesis has been previously proposed when studying a putative role of EMT process in circulating tumor cells (CSC) formation [10] and it appears today that EMT processes contribute at least in part to tumor heterogeneity [11] and CSC formation, supporting and validating the implication of EMT in MVI formation. In view of above understanding, our present study aimed to demonstrate the mechanism of MVI formation from the perspective of EMT process.

Currently, the exact mechanism of EMT progress remained largely unexplored. However, the critical role of some transcriptional factors (TFs) in the activation of EMT has been well documented. These TFs include the homeobox protein Goosecoid (Gsc)[12], the zinc-finger proteins Snai1 (Snail)and Snai2 (Slug) [13, 14], the basic helix-loop-helix protein Twist1 (Twist)[15], the forkhead box proteins FOXC1[16, 17] and FOXC2[18] and the zinc-finger, E-box-binding proteins Zeb1 and Sip1(Zeb2)[19, 20]. Additionally, a number of molecular processes have also been proposed to be engaged in order to initiate EMT. However, both developmental studies in various organisms and studies in tumor metastasis have yielded that TFs may serve as major signaling mediators in response to various inductive signals [8], For example, TGF- $\beta$ signaling, which is the best studied pathway responsible for inducing EMTs, represses the expression of E-cadherin through inducing snail, slug and twist[20,21]. Together, these studies have highlighted the major contribution of TFs during the execution of EMT program and subsequent metastatic cascade. Undoubtedly, the expression profile analysis and functional studies of these TFs maybe contribute to elucidate the possible involvement of EMT in MIV formation. In present study, we initially assessed by comparing the expression profiles of known EMT-related TFs between HCC tissues with and without MVI, and found that FOXC1 was the TF which presented prominently higher level in HCC tissues with MVI. We then explored the functional role of FOXC1 in regulating EMT, cell proliferation, metastatic potential, and VEGF-A expression for further understanding the mechanism underlying MVI formation of HCC.

\section{Material and method}

\section{Patient Samples}

50 HCC patients who underwent curative hepatic resection between March 2009 and June 2011 were recruited from our institute. The study was approved by the Ethics Committee of our hospital. Specimens were obtained immediately after surgical resection. None of patients were treated by any preoperative therapy such as TACE, percutaneous ethanol injection. Patients with macroscopic vascular invasion were excluded. There were 34 men and 16 women, ranging in age from 31 to 78 years, with a median age of 54 years. The presence of MVI was determined from histopathological reports stored in a prospectively maintained computerized clinical database. Tumor stage was defined according to American Joint Committee on Cancer/International Union Against Cancer tumor, node, metastasis (TNM) classification system [22]. Clinical data such as date of 
birth, sex, Child-Pugh stage at the time of surgery, date of surgery, serum a-fetoprotein, viral hepatitis status, tumor size, other content of histopathological reports were also extracted from the same database.

\section{Cell Lines}

All the Liver cancer cell lines were purchased from American Type Culture Collection (Manassas, USA) or Shanghai Institute of Cell Biology (Shanghai, China) and preserved in our institute. All of the cell lines were maintained in the recommended culture conditions and incubated at $37^{\circ} \mathrm{C}$ in a humidified environment containing $5 \% \mathrm{CO} 2$.

\section{Plasmids and Transfection}

The short-hairpin RNA (shRNA) sequences targeting human FOXC1 has been described [17] and a sequence nonspecific to any known gene were designed as a control by Genepharma (Shanghai, China), they were individually cloned into pGCsil/U6/Neomycin shRNA expression vector according to manufacturer's instruction. Full length human snail and twist gene were amplified from complementary DNA (cDNA) of human placental tissue and cloned into a pcDNA3.1(-) vector (Invitrogen, CA). Plasmids were transfected into cells with lipofectamine 2000 (Invitrogen, CA). Stable clone transfected with shRNA vector was selected using $800 \mathrm{ug} / \mathrm{ml}$ of neomycin (sigma, USA).

\section{Total RNA Extraction, Reverse Transcription and qRT-PCR}

Total RNA from tissues and cell was extracted using Trizol reagent (Invitrogen, CA). Reverse transcription for mRNAs was performed using the M-MLV Reverse Transcriptase (TaKaRa, Dalian, China). The cDNA template was amplified by qRT-PCR using the SYBR® Premix Dimmer Eraser kit (TaKaRa, Dalian, China). GAPDH was used as an internal control to normalize target mRNA level. qRT-PCR reactions were performed by the ABI7500 system (Applied Biosystems, CA). The relative expression fold change of mRNAs was calculated by the $2^{-\Delta \Delta \mathrm{Ct}}$ method. Primers were listed in Supplementary Material: Table S1.

\section{Western Blot Analysis}

Proteins from HCC cell lines were extracted with RIPA (Beyotime, Shanghai, China), separated by $8 \%-12 \%$ NUPAGE bis-tris Gel (Invitrogen, CA) and transferred onto polyvinylidene difluoride membranes. The following process was finished as decribed [23]. The primary antibodies were listed in Supplementary Material: Table S2.

\section{CCK-8 Assays}

Cell proliferation were examined using the CCK-8 cell counting kit (Dojindo Molecular Technologies, Japan) as manufacturer's instruction. The number of cells that were seeded onto 96-well plates was $5 \times 10^{3}$.

\section{Cell Cycle and Apoptosis Rate Analysis}

Cells stably transfected with shRNA were used for this analysis. For cell cycle analysis, the cells were fixed with ice-cold $75 \%$ ethyl alcohol at $4^{\circ} \mathrm{C}$ overnight and incubated with propidium iodide (BD Bioscience, CA) at $4^{\circ} \mathrm{C}$ in the dark for $30-60$ minutes. For apoptosis rate analysis, cells were incubated with Annexin V-FITC (BD Bioscience, CA) and propidium iodide for 5 minutes at $4^{\circ} \mathrm{C}$ in the dark. After staining, the cells were analyzed using a flow cytometer (CYTOMICS FC 500, Beckman Coulter, Miami, FL).

\section{Colony formation assay}

The Bel-7402 cells stably infected with shRNA $\left(1 \times 10^{4}\right)$ were plated in a $5 \mathrm{~cm}$ plate. The surviving colonies ( $>50$ cells) were counted with crystal violet staining after two weeks of culture. Colony-forming efficiency (CFE \%) was defined as the ratio of the number of colonies formed in culture to the number of cells inoculated. This experiment was performed in triplicate.

\section{Invasion assay}

Cell-invasion assay was performed using a transwell assay (Millipore, Billerica, MA). The details of this method have been described [23]. The number of cells placed in the upper chamber was $5 \times 10^{5}$. The stained cells were counted under an inverted microscope (5 fields per membrane). Each experiment was performed in triplicate.

\section{Wound Healing Assay}

Cells $\left(5 \times 10^{6}\right)$ were implanted onto a 6-well plate. When cells grew to $80 \%$ confluence, the cell monolayer was wounded with a $10 \mu \mathrm{L}$ plastic pipette tip. The remaining cells were incubated at normal culture medium. At the indicated times, migrating cells at the wound front were photographed and the percentage of the cleaned area at each time point compared with time 0 was measured using Image-Pro Plus version 6.2 software.

\section{F-actin Immunofluorescence}

Cells $\left(5 \times 10^{3}\right)$ were implanted onto a cell culture dish (NEST Biotech, Hong Kong) for 24 hours. Cells were fixed with paraformaldehyde for 30 minutes, then permeabilized with $0.1 \%$ Triton X-100 for $5 \mathrm{~min}$ 
at room temperature and thereafter incubated with Atto 655-phalloidin (sigma, USA) in the dark for 15 min at room temperature. Fluorescence images were photographed with both microscope fluorescence (Olympus LX81, Japan) and confocal microscopy(Leica DMIRE2, Germany)

\section{Confocal Immunofluorescent Analysis}

For indirect immunofluorescence, cells were similarly processed as described above, and the primary antibodies were added after permeabilization. After incubation in the dark for 24 hours at $4^{\circ} \mathrm{C}$, cells were incubated with alexa flours $488 \mathrm{TgG}$ donkey anti-mouse or anti-rabbit (1:500, Invitrogen, USA) for an hour at room temperature, $\mathrm{Nu}-$ clei were stained with propidium iodide for 5 minutes when necessary. Fluorescence images were photographed with a confocal microscopy

\section{Statistical Analysis}

Comparisons of continuous data were analyzed by the independent $t$ test between the 2 groups, whereas categorical data was analyzed by the chi-square test. All statistical analyses were performed using SPSS for Windows v.16.0 (SPSS, Chicago, IL) and GraphPad Prism 5.0 (GraphPad Software, La Jolla, CA). $\mathrm{P}<0.05$ was considered statistically significant.

\section{Results}

\section{The expression of EMT-related genes in various HCC tissues and cell lines}

To elucidate the conjunction between EMT and MVI formation, we first concurrently evaluated the expression profiles of EMT-promoting TFs (Snail, Slug, Twist, Goosecoid, Zeb1, Zeb2, FOXC1, FOXC2) and EMT-regulated genes (E-cadherin, $\mathrm{N}$-cadherin, and Vimentin) in HCC tissues. We found that the tissue with MVI expressed higher levels of FOXC1, Snail, Vimentin and lower levels of E-cadherin when compared with the tissue without MVI (all $P<0.05$, Fig.1A). Especially, among these up-regulated TFs, the FOXC1 discrepancy was the most significant, increasing by more than 2.5 folds. Thus, we hypothesized that FOXC1 might act as a major modulator of EMT in HCC tissue and a candidate mediator of MVI formation.
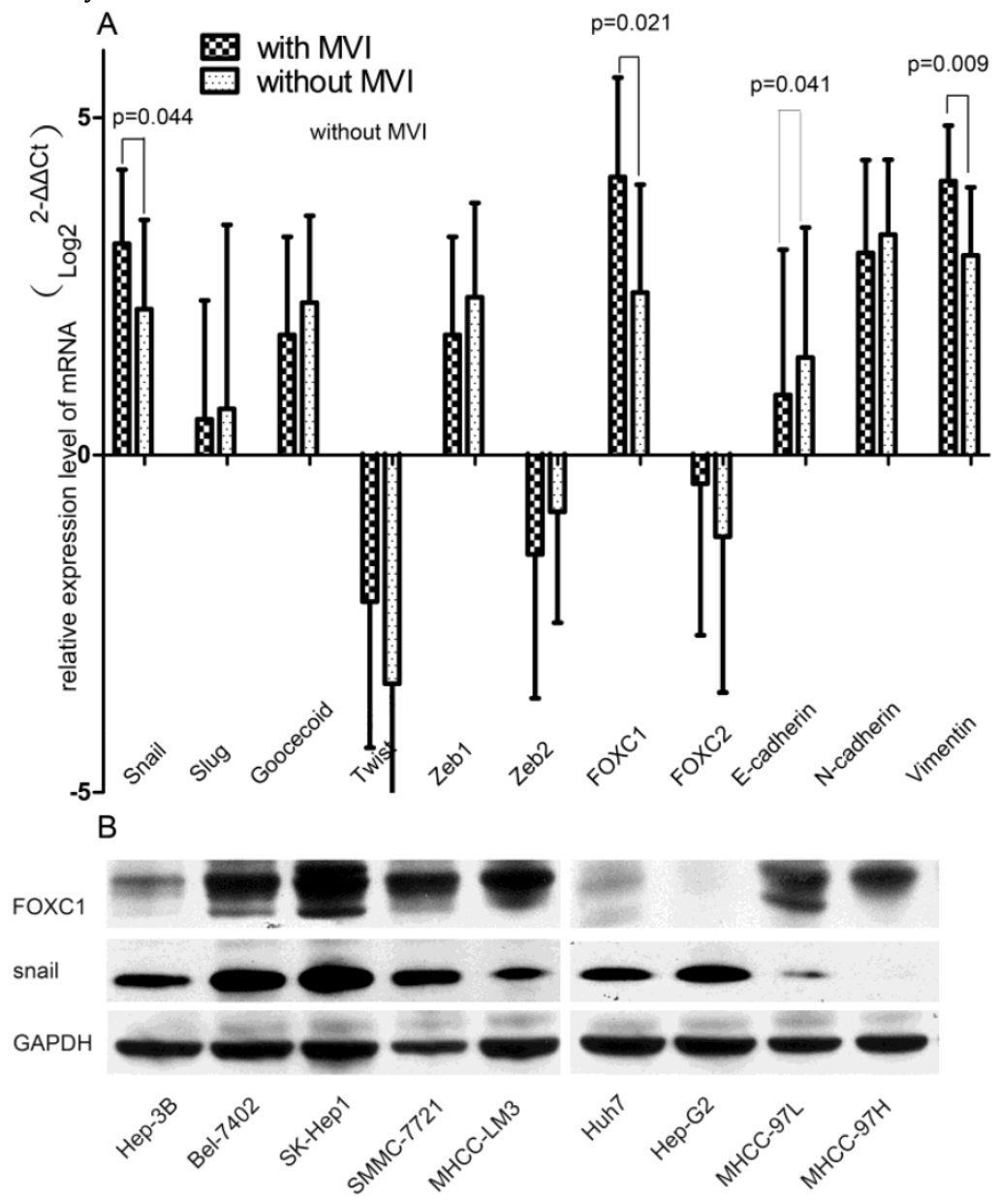

C

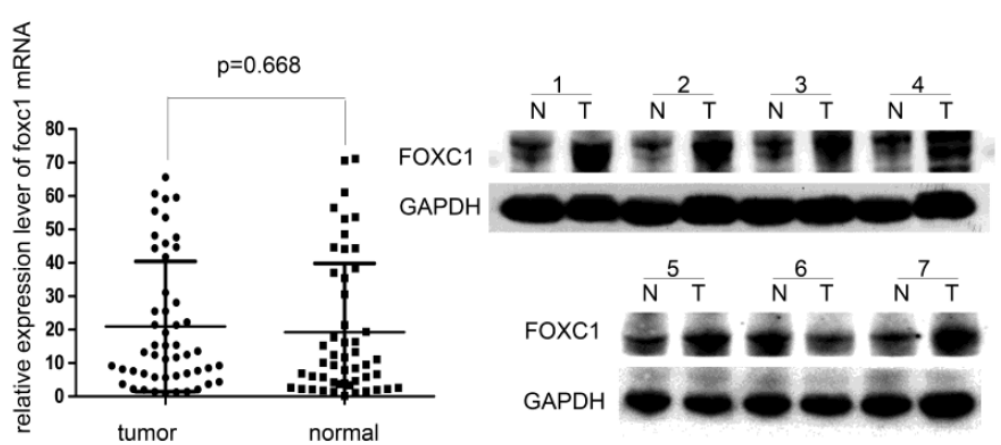


Tabel I: the relationship between FOXCI expression and clinicopathological features of 50 patients with hcc.

\begin{tabular}{|c|c|c|c|c|}
\hline \multirow{2}{*}{$\begin{array}{l}\text { clinicopathological } \\
\text { variables }\end{array}$} & \multirow{2}{*}{$\begin{array}{l}\text { Number of } \\
\text { each group }\end{array}$} & \multicolumn{2}{|c|}{ FOXC1 expression } & \multirow{2}{*}{$\begin{array}{l}\mathrm{P} \\
\text { value }\end{array}$} \\
\hline & & high & low & \\
\hline All cases & 50 & 27 & 23 & \\
\hline \multicolumn{5}{|l|}{ Age(years) } \\
\hline$\leqq 50$ & 9 & 4 & 5 & 0.79 \\
\hline$>50$ & 41 & 23 & 18 & \\
\hline \multicolumn{5}{|l|}{ Tumor number } \\
\hline Solitary & 44 & 24 & 20 & 1.0 \\
\hline Multiple & 6 & 3 & 3 & \\
\hline \multicolumn{5}{|l|}{ Gender } \\
\hline Male & 34 & 16 & 18 & 0.151 \\
\hline female & 16 & 11 & 5 & \\
\hline \multicolumn{5}{|l|}{$\begin{array}{l}\text { Size of largest tu- } \\
\text { mor, }(\mathrm{cm})\end{array}$} \\
\hline$<5$ & 25 & 13 & 12 & 0.777 \\
\hline$\geqq 5$ & 25 & 14 & 11 & \\
\hline \multicolumn{5}{|c|}{$\begin{array}{l}\text { Histological differen- } \\
\text { tiation }\end{array}$} \\
\hline Well & 5 & 1 & 4 & $0.034 *$ \\
\hline moderate & 21 & 21 & 10 & \\
\hline poor & 14 & 5 & 9 & \\
\hline \multicolumn{5}{|l|}{ Liver cirrosis } \\
\hline yes & 39 & 22 & 17 & 0.520 \\
\hline No & 11 & 5 & 6 & \\
\hline \multicolumn{5}{|l|}{ HBV } \\
\hline positive & 37 & 22 & 15 & 0.191 \\
\hline negative & 13 & 5 & 8 & \\
\hline \multicolumn{5}{|l|}{$\begin{array}{l}\text { Serum AFP val- } \\
\text { ue(ug/L) }\end{array}$} \\
\hline$<25$ & 17 & 9 & 8 & 0.914 \\
\hline$\geqq 25$ & 33 & 18 & 15 & \\
\hline \multicolumn{5}{|l|}{ MVI } \\
\hline Yes & 16 & 12 & 4 & $0.041 *$ \\
\hline No & 34 & 15 & 19 & \\
\hline \multicolumn{5}{|l|}{ Microsatellite lesion } \\
\hline Yes & 5 & 1 & 4 & 0.250 \\
\hline No & 45 & 26 & 19 & \\
\hline \multicolumn{5}{|l|}{$\begin{array}{l}\text { Invasion of contigu- } \\
\text { ous organs }\end{array}$} \\
\hline Yes & 6 & 5 & 1 & 0.271 \\
\hline No & 44 & 22 & 22 & \\
\hline \multicolumn{5}{|l|}{ CK19 } \\
\hline positive & 21 & 13 & 8 & 0.34 \\
\hline negative & 29 & 14 & 15 & \\
\hline \multicolumn{5}{|l|}{ CK 34} \\
\hline positive & 22 & 15 & 7 & 0.075 \\
\hline negative & 28 & 12 & 16 & \\
\hline
\end{tabular}

$* \mathrm{P}<0.05$
We further tested the expression of FOXC1 in 9 HCC cells lines with different metastatic abilities. FOXC1 expression increased approximately in parallel with the metastatic potential of HCC cell lines. Compared with no-metastatic cells (Huh7, HepG2 and Hep-3B), the other cancer cell lines (Bel-7402, SK-Hep1, SMMC-7721, MHCC-97L, MHCC-97H, and MHCC-LM3) consistently expressed higher levels of FOXC1 (Fig.1B).

\section{Expression of FOXCI in HCC tissue and its relationship to clinicopathological parameters}

We evaluated the FOXC1 expression using qRT-PCR on 50 paired HCC surgical tissues (tumor tissues and matched adjacent non-tumor liver tissues). Our result showed that nearly $54 \%$ of primary HCC tumors expressed higher level of FOXC1 compared with the matched adjacent non-tumor liver tissues (Fig. 1C), however, the difference was not significant $(P=0.668)$.We next evaluated the relationship between FOXC1 expression and various clinicopathological parameters. FOXC1 expression was significantly correlated with MVI incidence and histological differentiation $(P<0.05$, Table 1$)$. There was no statistically significant difference in FOXC1 expression by age, gender, liver cirrhosis, HBV, CK19, CK34, serum AFP, and Microsatellite lesion (Table 1). To investigate whether FOXC1 was also over-expressed at the protein level in tumor tissue with MVI, western-blot was performed on 15 HCC clinical samples with MVI. As shown in Figure 1D, FOXC1 protein expression was higher in the tumors, consistent with the results of real-time quantitative PCR.

\section{FOXCI Knockdown Depressed Mesenchymal Differentiation}

To determine the pathological role of FOXC1 in EMT processes of HCC cells, we used knockdown strategy with shRNA in Bel-7402 cell and SK-Hep1 cells, which express high level of FOXC1. FOXC1 expression in the transfected cells was confirmed by qRT-PCR and western-blot (Fig. 2A, 2B). We proceeded to test some phenotypic markers with western-blot and found decreased expression of FOXC1 was correlated with repressed expression of mesenchymal markers such as Vimentin and N-cadherin. The expression of epithelial cell-specific protein, however, was only partially affected. Concretely, the expression of E-cadherin, a central marker of epithelial cell phenotype, was not consistently reduced with the down-expression of FOXC1 (Fig. 2C), Meanwhile, western-blot examination showed that some other epithelial cell-specific proteins such as ZO-1 and claudin-1 were significantly elicited. Some above re- 
sults were confirmed by confocal microscopy examination in Bel-7402 cells. Moreover, the redistribution of $\beta$-catenin without significant change of content was also observed (Fig. 2C, 2D), reflecting the reorganization of intercellular junctions
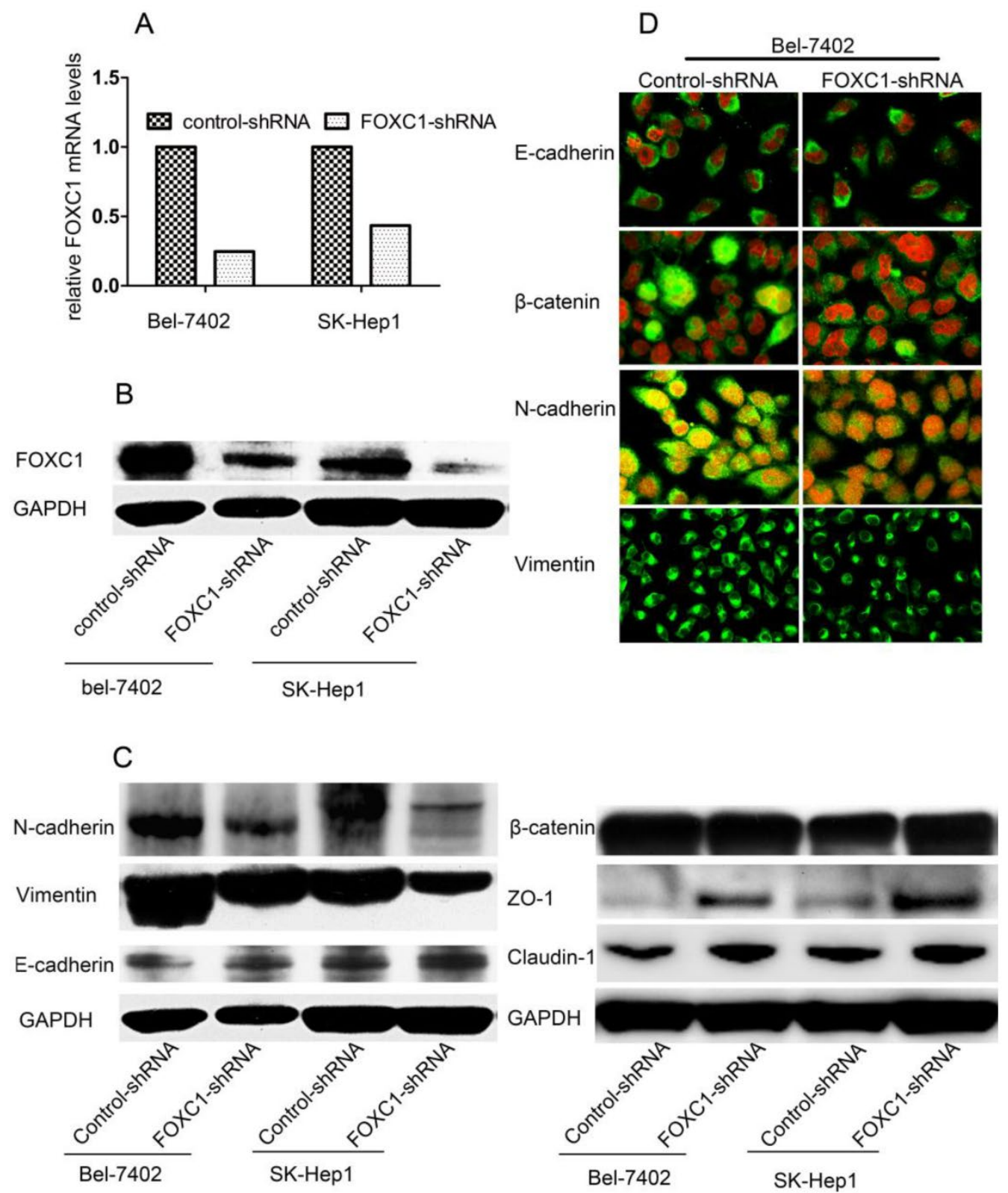

Figure 2: Effect of FOXCI knockdown on the expression of epithelial and mesenchymal markers. (A) Knockdown of FOXCI mRNA expression in Bel-7402 and SK-HepI through shRNA plasmid transfection was confirmed by qRT-PCR (B) Knockdown of FOXCI protein in Bel-7402 and SK-Heplafter transfection was confirmed by Western-blot. (C) Western-blot analysis of phenotypic markers including E-cadherin, Vimentin, N-cadherin, ZO-I, Claudin-I and $\beta$-catenin in cells transfected with control-shRNA and FOXCI-shRNA. (D) Confocal microscopy analysis of phenotypic marker including E-cadherin, $\mathrm{N}$-cadherin, Vimentin,and $\beta$-catenin. The green signal represents the staining of corresponding protein, and the red signal represents the nuclear DNA staining by DAPI. 


\section{Interaction between FOXCI and EMT-inducer}

To further understand the function of FOXC1 in inducing EMT, we tested how down-expression of FOXC1 affected the expression of other TFs. We used western-blot to test the expression level of snail, twist, slug in Bel-7402 and SK-Hep1 cell after FOXC1 knockdown and confirmed none of these TFs was consistently depressed in respond to inhibition of FOXC1 (Fig. 3A). We further studied whether other TFs modulated the expression of FOXC1. pcDNA3.1(-)-snail, pcDNA3.1(-)-twist or control vector were transfected into Huh7 cells, which expresses low endogenous FOXC1 level, and western-blot was used to confirm the validity of these transfections (Fig. 3B). We observed that ectopic expression of both snail and twist could induce the expression of FOXC1 (Fig. 3C). Moreover, the expression level of FOXC1 in Huh7 cells transfected with pcDNA3.1(-)-snail was relatively higher.

TGF- $\beta 1$ is one of the most potent EMT-inducer present in tumor microenvironment. Indeed, treatment certain cell with TGF- $\beta 1$ for a period 12 day can induce an EMT and the expression of some EMT-inducing TFs [18]. Here, we tested the expression of FOXC1 by qRT-PCR in Huh7 cell after treatment with TGF- $\beta 1$ for 14 days. The elevated expression of FOXC1 was firstly detected on day 8 after TGF- $\beta 1$ treatment, and increased by more than 5 folds on day 14 (Fig. 3D). In addition, we detected the expression of TGF- $\beta 1, \beta 2, \beta 3$ mRNA in Bel-7402 cell after FOXC1 knockdown to determine whether FOXC1 affected, in turn, the expression TGF- $\beta$. We found none of these isomers expression was altered in this condition (Fig. 3E), suggesting that FOXC1 may locate in the TGF- $\beta 1$-induced downstream.
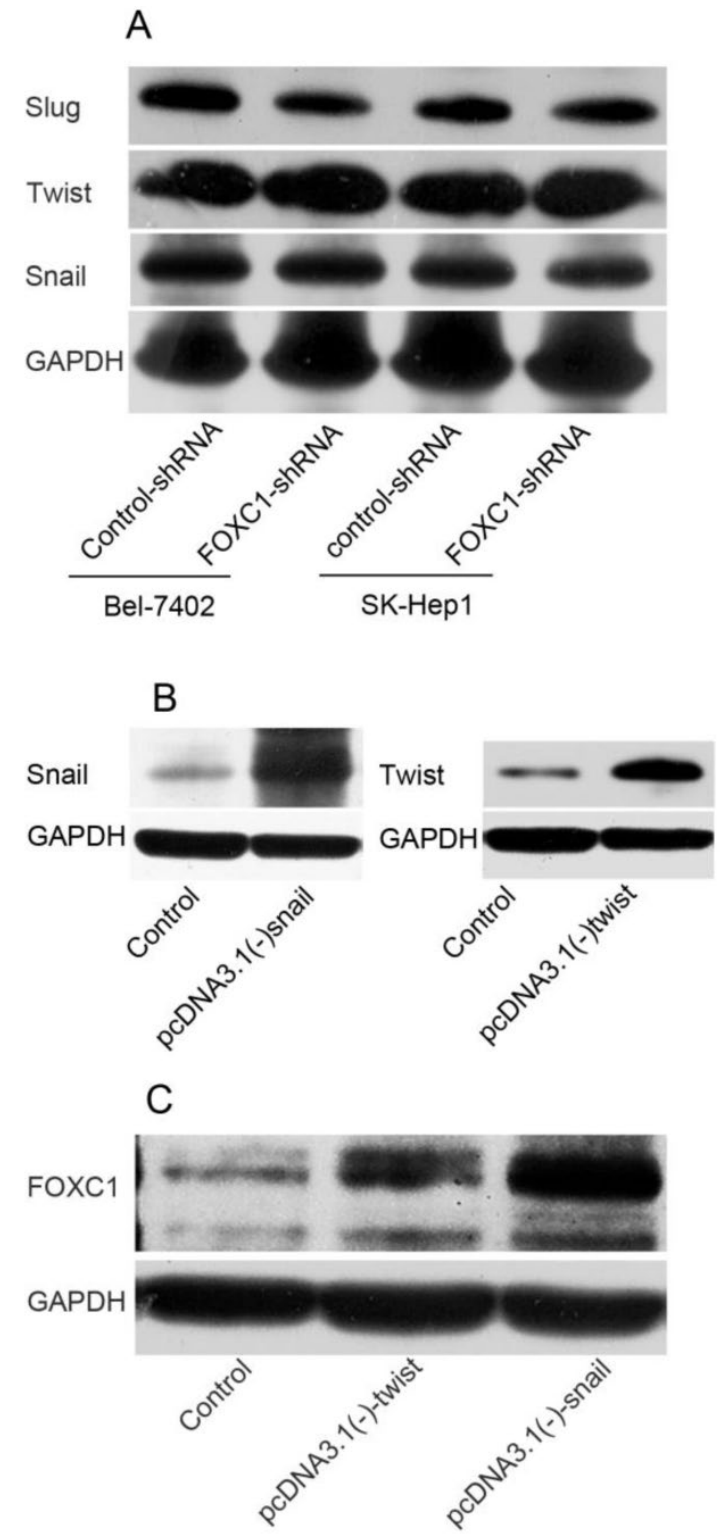

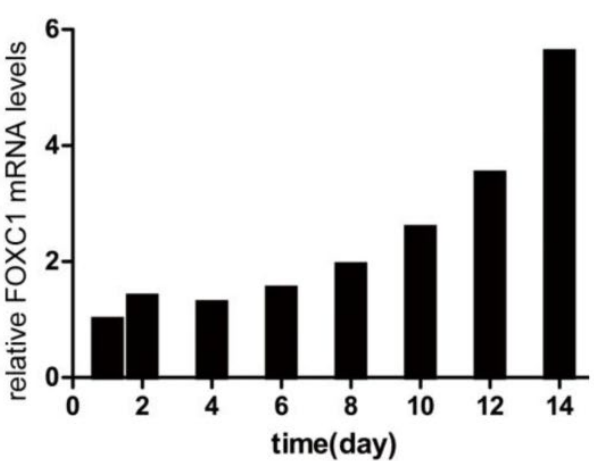

E

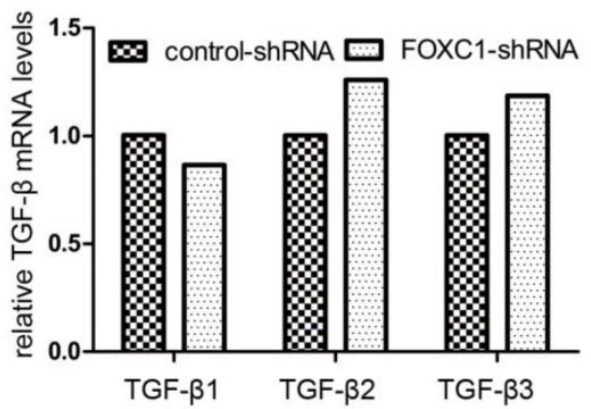

Figure 3: Interaction between FOXCI and other EMT-regulator. (A) Expression of snail, slug and twist protein in Bel-7402 after transfected with control-shRNA or FOXCI-shRNA was examined by western-blot. (B) Over-expression of snail and twist in Huh7 cells after transfected by vector or control was confirmed by western-blot. (C) Expression of $\mathrm{FOXCl}$ protein in the Huh7 cell ectopically expressing either a control vector or EMT-inducing genes was examined by western-blot. (D) Expression of FOXCl mRNA in huh7 cells treated with TGF- $\beta$ I was shown for the indicated number of days..(E) Relative expression level of TGF- $\beta$ I, $\beta 2, \beta 3$ mRNA in Bel-7402 transfected with control-shRNA or FOXCI-shRNA was measured by $\mathrm{qRT}$-PCR. 


\section{FOXCI Knockdown Inhibited the Viability of Bel-7402 Cell}

To explore the effect of FOXC1 knockdown on tumor cell growth, we used CCK-8 test to measure cellular viability within 7 days after FOXC1 knockdown and found FOXC1 down-regulation sufficiently

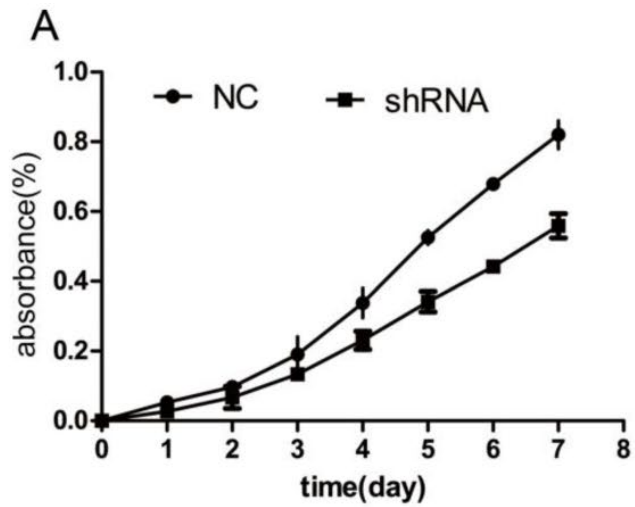

B

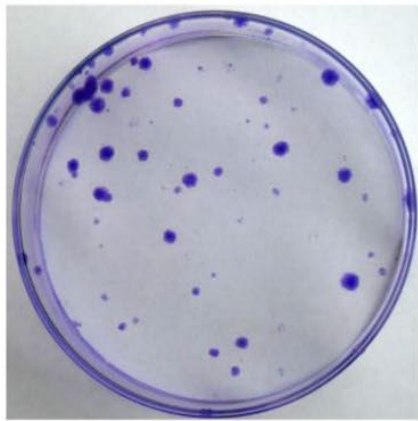

control-shRNA

C
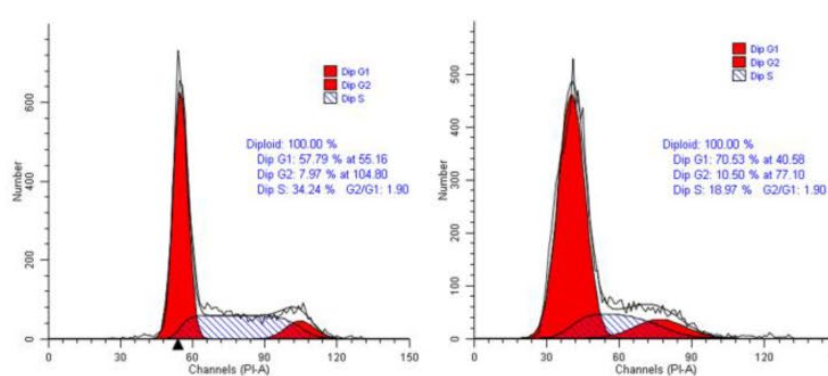

D

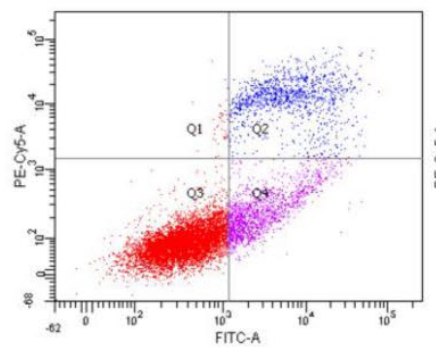

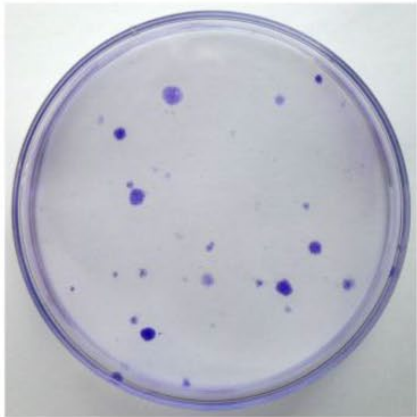

FOXC1-shRNA

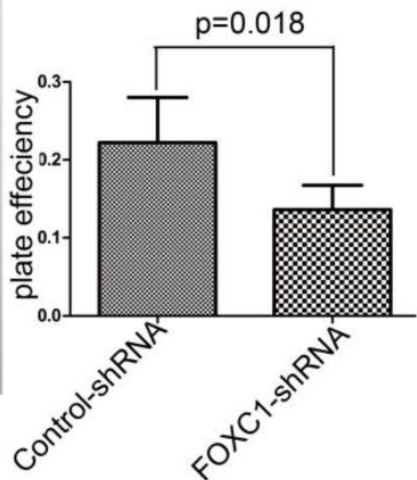

m control-shRNA
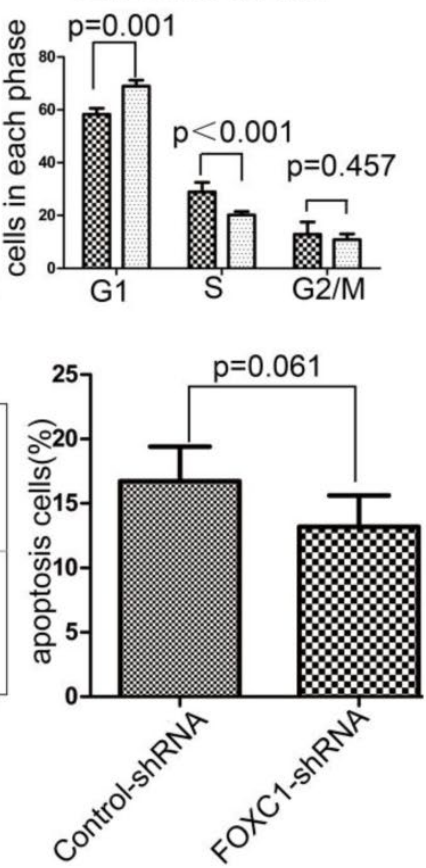

decreased the cell viability (Fig. 4A). Next, we observed a significant reduction in colony formation ability in cells transfected with FOXC1-shRNA $(P<$ 0.05 , Fig. 4B). These observations collectively suggest that FOXC1 depression may regulate cell cycle or apoptosis. Then, we proceeded to use flow cytometer to investigate apoptosis and cell cycle, and found that

FOXC1 knockdown induced G1 phase arrest $(P<$ 0.05, Fig. 4C). In contrast, no significant change of apoptosis rate was observed $(P>0.05$, Fig. $4 \mathrm{D})$, suggesting that FOXC1 knockdown inhibited the viability of Bel-7402 cell through inducing G1 phase arrest rather than apoptosis.

Figure 4: Effect of FOXCI knockdown on HCC cell viability. (A) CCK-8 analysed the growth curve of Bel-7402 cell transfected with control-shRNA or FOXCI-shRNA. (B) Colony formation assay evaluated the cell growth after tranfection. (C) The cell cycle of Bel-7402 after transfection was valuated with flow cytometer. (D) The apoptosis rate of Bel-7402 after transfection was counted with flow cytometer. 
Figure 5: Effect of FOXCI knockdown on cellular ability of migration and invasion. (A) Transwell invasion assays of Bel-7402 cells after transfected with control-shRNA or FOXCI-shRNA. Images are shown on the left (magnification: $\times 100$ ), and the quantification of 5 randomly selected fields was shown on the right. (B) Wound healing assay of Bel-7402 cells after transfection. Images are shown on the left (magnification: $\times 100$ ), and the cleaned area was measured and plotted as the percentage of the original time point $(0$ hour). (C) F-actin was stained with Atto 655-phalloidin to evaluate cytoskeleton and photographed with both microscope fluorescence (left, magnification: $\times 200$ ) and confocal microscopy (right, magnification: $\times 1200$ ). (D) Western-blot examined the expression levels of some MMPs and VEGF-A protein in the Bel-7402 cell after tranfection.

\section{FOXCI Silencing Suppressed Migration and Invasiveness in Bel-7402 Cell}

We determined whether down-regulation of FOXC1 rendered altered ability of migration and invasiveness using wound healing assay and transwell assay. In transwell assay, FOXC1-shRNA Bel-7402 cells, migrated into the lower compartment of the migration chamber, were significantly less than control-shRNA Bel-7402 cells $(P<0.05$, Fig. 5A). In wound healing assay, microscopic examination at 12 and $24 \mathrm{~h}$ revealed a significant delay in the wound closure of Bel-7402 cells after FOXC1 silencing $(P<$ 0.05, Fig. 5B).

Actin cytoskeleton organization and polymerization have a well described role in cell migration. In present study, we used Atto 655-Phalloidin to mark distribution and dynamic changes of the actin cytoskeleton in cells. The decreased fluorescence intensity, which reflecting F-actin content, was observed in FOXC1-shRNA cells (Fig. 5C). Additionally, inhibition of FOXC1 expression led the stress fibers to be disassembled and disrupted into short fragments with irregular pattern, accompanied by cell rounding (Fig. $5 C)$.

\section{FOXCI Silencing Depressed the Expression of Certain MMPs Proteins and VEGF-A}

Various MMPs proteins seen to be capable of prompting cancer cell migration ability, we accordingly determined the effect of FOXC1 knockdown on certain MMPs expressions. Our western-blot results confirmed that the expression of MMP1, MMP2, MMP7 and MMP9 were consistently reduced in respond to FOXC1 silencing, suggesting that these four MMPs could be involved in FOXC1-related elevation of migratory potency. In contrast, MMP3, MMP13 were not down-regulated by FOXC1 knockdown (Fig.
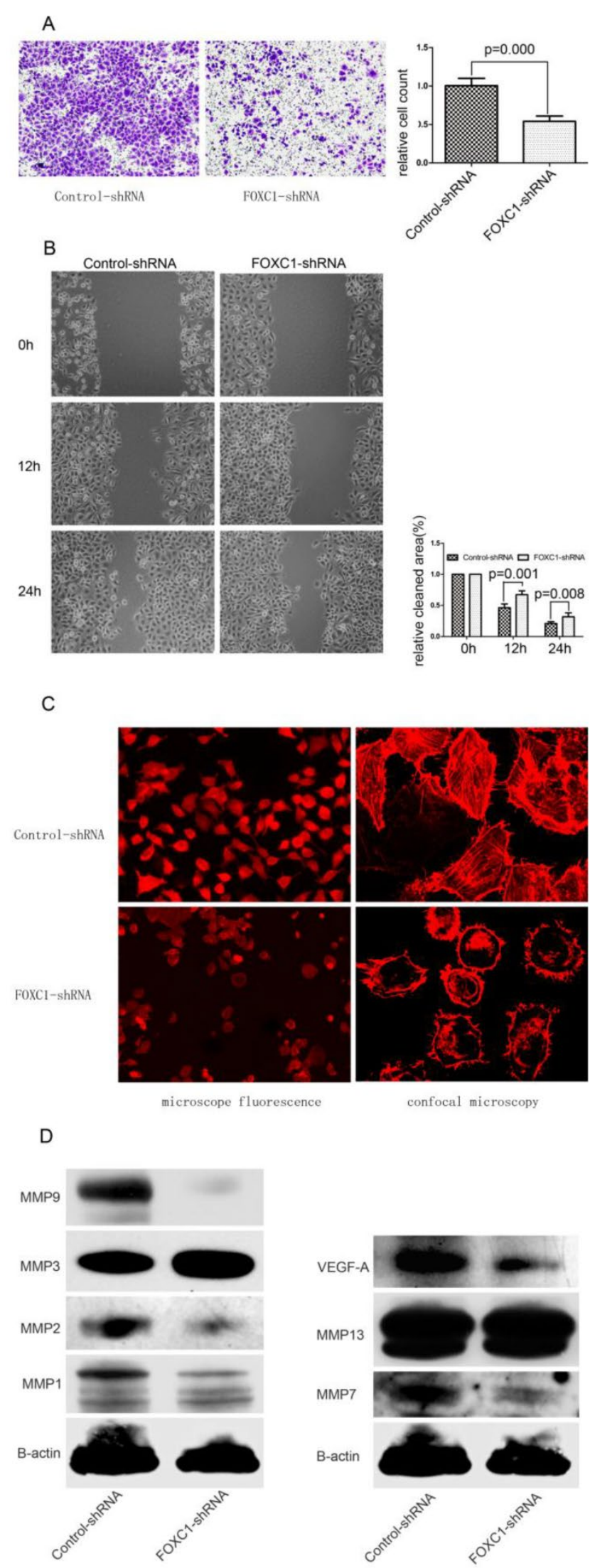
5D). In addition, we also evaluated the alternation of VEGF-A expression, whose predictive role in angiogenesis has been widely understood, and revealed that VEGF-A expression level was significantly decreased after FOXC1 silencing (Fig. 5D), indicating a possible contribution of FOXC1 to tumor angiogenesis.

\section{Discussion}

In this study, we identified the over-expression of FOXC1 as a prominent molecular characteristic of the HCC with MVI. FOXC1 protein belongs to the Forkhead Box (FOX) containing transcription factor family. These proteins are characterized by a conserved 110 amino acid DNA-binding domain known as a forkhead domain (FHD). FOX proteins are key regulators of diverse cellular functions, including the development of many organ systems, energy homeostasis and oncogenesis [24]. The FOXC1 gene is extensively expressed in mesenchyme and its protein product has been widely reported to participate in abroad range of developmental processes [25]. Relatively speaking, the role of FOXC1 in cancer progression receives less attention and has mainly been investigated in breast cancer. Bloushtain-Qi et al reported that expression of FOXC1 in MCF12A cells resulted in increased cell migration [16]. Notably, over-expression of the FOXC1 was identified as a consistent feature of basal-like breast cancer (BLBC) compared with other molecular subtypes of breast cancer, and elevated FOXC1 expression predicted poor overall survival along with a higher incidence of brain metastasis [17]. As far as HCC is concerned, the clinical significance of FOXC1 remained unclear. Here, we reported that the over-expression of FOXC1 gene was associated with MVI formation and histological differentiation. Though more follow-up data, such as overall and disease-free survivals, was needed for further assessment, the clinicopathological significance of FOXC1 gene in HCC could be preliminarily evaluated.

As anticipated, present study showed that suppressed FOXC1 expression led to a reversion of EMT progress, characterized by decreased expression of mesenchymal markers (Vimentin, N-catenin), translocation of $\beta$-catenin, and increased expression of some epithelial markers (ZO-1 and Claudin-1). However, epithelial traits were only partially impacted in this condition, and E-cadherin remained unchanged in both expression level and distribution. This model was consistent with a previous observation that the FOXC1 gene is widely expressed in mesenchyme and its loss or mutation leads to mesenchymal abnormality due to loss of mesen- chyme-secreted signaling molecules [26]. The involvement of FOXC1 in regulating tumor EMT has been previously described in limited tumor, mainly in breast cancer. Nevertheless, Ray et al [17] reported that ectopic expression of FOXC1 in breast cancer cell led to the complete conversion of EMT including down-regulated expression of E-cadherin. Wang et al also reported FOXC1 regulated complete EMT progress by activating NF-kappaB signaling [27].Their observations contrasted sharply with our result regarding FOXC1 role of modulating E-cadherin expression. This phenomenon suggested that the functional role of FOXC1 might have tissue specificity. Interestingly, FOXC2, another protein of FOXC class, has also been reported to be capable of modulating only mesenchymal half of EMT transdifferentiation [18]. Since FOXC1 was not sufficient to induce entire EMT process on its own, we further investigated its role in regulating EMT via TFs interaction. We observed that none of snail, slug, twist or TGF- $\beta$ was down-regulated by FOXC1 knockdown. In contrast, expression of FOXC1 was elevated consistently in response to over-expression of snail, twist and TGF- $\beta 1$. Taken together, we proposed that FOXC1 might operate the downstream of other EMT regulators and induce the mesenchymal part of their EMT program. Additionally, the tissues with MVI co-expressed higher levels of FOXC1and Snail when compared with the tissues without MVI, a positive correlation of expression with FOXC1 could be only established for snail and, not for twist, in vivo. This phenomenon can be explained by the complexity of EMT-regulating network.

Furthermore, present experiment determined that suppressed expression of FOXC1 in Bel-7402 cells rendered their lower invasion and migration. Previous findings have suggested that FOXC1 endows tumor cells with enhanced invasion through increased expression of different MMPs[28]. We thus tested the expression level of different MMPs in FOXC1-delepted cell, and revealed that the MMP1, MMP2, MMP7, MMP9 were simultaneously down-regulated in FOXC1 depressed cell, indicating the role of MMPs in FOXC1-induced enhancement of migration and invasion. Besides MMPs aberrant expression, our results showed that cytoskeleton modification might also contribute to decreased migration and invasion in FOXC1-silencing cells. Intermediate filaments (IFs) are one of major cytoskeletal systems of vertebrate cells and the mesenchymal IF network is primarily constituted of Vimentin. Extensive literatures have identified many EMT-related TFs such as snail, slug and twist to induce the Vimentin expression. Similarly, our present studied identified FOXC1 
sufficient to regulate the Vimentin expression. Besides the IF network, actin cytoskeleton organization and polymerization have a coordinately role in cell migration. We also observed that down-regulation of FOXC1 was associated with depolymerization of F-actin cytoskeleton and diminution of F-actin content accompanied by morphological changes, indicating the repressed cell mobility. Although the regulatory mechanism of actin cytoskeleton reorganization induced by FOXC1 was waiting to be elucidated, some molecular mediators which play a central role in modulating the actin cytoskeleton, such as Rho GTPase and integrins [29] have been shown to be closely regulated during EMT processes.

Tumor cell invasion itself is not sufficient to produce MVI formation. Stimulation of angiogenesis and intravasation of tumor cell are important aspects. Nowadays, extensive documents have explored the EMT implication in regulation of angiogenesis and revealed that EMT can promote angiogenesis by modulating pro-angiogenic factors such as VEGF-A. Our present study also revealed that down-regulation of FOXC1 was associated with repressed expression of VEGF-A. In addition, MMP1 and MMP4 expressions, which have definite role in enhancing angiogenesis, were observed to be down-regulated by FOXC1 knockdown. These finding indicated the possible involvement of FOXC1 in promoting angiogenesis. Consistence with our result, FOXC1 expression has been reported to influence the proliferation and differentiation of endothelial development $[30,31]$. As for intravasation of tumor cell, the participation of EMT-related genes in the regulation of transendothelial migration is nowadays becoming clear. For example, N-cadherin has been reported to play a major role in transendothelial migration by contributing to heterotypic contacts between endothelial cells and melanoma cells. $\beta$-catenin translocation from cell-cell adhesion contact to nucleus also contribute to enhance transendothelial cell migration[32]. In present study, both decreased expression of $\mathrm{N}$-cadherin and redistribution of $\beta$-catenin were observed. These data provided the possibility that FOXC1 might participate the regulation of intravasation and further favored the MVI generation. But this hypothesis requires further analysis in mouse models.

In summary, high FOXC1 expression was correlated with occurrence of MVI formation in HCC patients and suppression of endogenous FOXC1 expression resulted in a partial conversion of EMT progress in HCC cell lines, mainly influencing their mesenchymal component. Moreover, inhibition of FOXC1 expression in HCC cells represents a promising option to prevent HCC metastasis through recon- struction of cellular behavior including proliferation, migration and invasion, angiogenesis and intravasation.

\section{Supplementary Material}

Table S1. List of proteins tested by antibodies and characteristics of the corresponding antibodies used. Table S2. Sequence of the oligonucleotides for plasmid construct-making and real-time PCR. http://www.biolsci.org/v08p1130s1.pdf

\section{Abbreviations}

HCC: Hepatocellular carcinoma; MVI: Microvascular invasion; TFs: Transcriptional factors; EMT: Epithelial-Mesenchymal Transition

\section{Acknowledgment}

This study was supported by the National S\&T Major Project (No. 2012ZX10002-017), NSFC for Innovative Research Group (81121002) and National Basic Research Program of China (973 Program) (No.2009CB522403). We thank the surgeons and nurses who kindly facilitate the recruitment and collection of patient information.

\section{Competing Interests}

The authors have declared that no competing interest exists.

\section{References}

1. Hadziyannis SJ, Tassopoulos NC, Heathcote EJ, et al. Long-term therapy with adefovir dipivoxil for HBeAg-negative chronic hepatitis B for up to 5 years. Gastroenterology. 2006; 131: 1743-1751.

2. Poon RT, Fan ST, Tsang FH, et al. Locoregional therapies for hepatocellular carcinoma: a critical review from the surgeon's perspective. Ann Surg. 2002; 235: 466-486.

3. Hu J, Wang Z, Fan J, et al. Genetic variations in plasma circulating DNA of HBV-related hepatocellular carcinoma patients predict recurrence after liver transplantation. PLoS One. 2011; 6: e26003.

4. Roayaie S, Frischer JS, Emre SH, et al. Long-term results with multimodal adjuvant therapy and liver transplantation for the treatment of hepatocellular carcinomas larger than 5 centimeters. Ann Surg. 2002; 235: 533-539.

5. Yao FY, Ferrell L, Bass NM, et al. Liver transplantation for hepatocellular carcinoma: comparison of the proposed UCSF criteria with the Milan criteria and the Pittsburgh modified TNM criteria. Liver Transpl. 2002; 8: 765-774.

6. Kurrey NK, Jalgaonkar SP, Joglekar AV, et al. Snail and slug mediate radioresistance and chemoresistance by antagonizing p53-mediated apoptosis and acquiring a stem-like phenotype in ovarian cancer cells. Stem Cells. 2009; 27: 2059-2068.

7. Taube JH, Herschkowitz JI, Komurov K, et al. Core epithelial-to-mesenchymal transition interactome gene-expression signature is associated with claudin-low and metaplastic breast cancer subtypes. Proc Natl Acad Sci U S A. 2010; 107: 15449-15454.

8. Yang J, Weinberg RA. Epithelial-mesenchymal transition: at the crossroads of development and tumor metastasis. Dev Cell. 2008; 14: 818-829.

9. Kalluri R, Weinberg RA. The basics of epithelial-mesenchymal transition. J Clin Invest. 2009; 119: 1420-1428.

10. Bonnomet A, Brysse A, Tachsidis A, et al. Epithelial-to-mesenchymal transitions and circulating tumor cells. J Mammary Gland Biol Neoplasia. 2010; 15: 261-273. 
11. Klymkowsky MW, Savagner P. Epithelial-mesenchymal transition: a cancer researcher's conceptual friend and foe. Am J Pathol. 2009; 174: 1588-1593.

12. Hartwell KA, Muir B, Reinhardt F, et al. The Spemann organizer gene, Goosecoid, promotes tumor metastasis. Proc Natl Acad Sci U S A. 2006; 103: 18969-18974.

13. Batlle E, Sancho E, Franci C, et al. The transcription factor snail is a repressor of E-cadherin gene expression in epithelial tumour cells. Nat Cell Biol. 2000; 2: 84-89.

14. Hajra KM, Chen DY, Fearon ER. The SLUG zinc-finger protein represses E-cadherin in breast cancer. Cancer Res. 2002; 62: 1613-1618.

15. Yang J, Mani SA, Donaher JL, et al. Twist, a master regulator of morphogenesis, plays an essential role in tumor metastasis. Cell. 2004; 117: 927-939.

16. Bloushtain-Qimron N, Yao J, Snyder EL, et al. Cell type-specific DNA methylation patterns in the human breast. Proc Natl Acad Sci U S A. 2008; 105: 14076-14081.

17. Ray PS, Wang J, Qu Y, et al. FOXC1 is a potential prognostic biomarker with functional significance in basal-like breast cancer. Cancer Res. 2010; 70: 3870-3876.

18. Mani SA, Yang J, Brooks M, et al. Mesenchyme Forkhead 1 (FOXC2) plays a key role in metastasis and is associated with aggressive basal-like breast cancers. Proc Natl Acad Sci U S A. 2007; 104: 10069-10074.

19. Eger A, Aigner K, Sonderegger S, et al. DeltaEF1 is a transcriptional repressor of E-cadherin and regulates epithelial plasticity in breast cancer cells. Oncogene. 2005; 24: 2375-2385.

20. Comijn J, Berx G, Vermassen P, et al. The two-handed E box binding zinc finger protein SIP1 downregulates E-cadherin and induces invasion. Mol Cell. 2001; 7: 1267-1278.

21. Li H, Wang H, Wang F, et al. Snail involves in the transforming growth factor beta1-mediated epithelial-mesenchymal transition of retinal pigment epithelial cells. PLoS One. 2011; 6: e23322.

22. Lu W, Dong J, Huang Z, et al. Comparison of four current staging systems for Chinese patients with hepatocellular carcinoma undergoing curative resection: Okuda, CLIP, TNM and CUPI. J Gastroenterol Hepatol. 2008; 23: 1874-1878.

23. Cheng J, Xie HY, Xu X, et al. NDRG1 as a biomarker for metastasis, recurrence and of poor prognosis in hepatocellular carcinoma. Cancer Lett. 2011; 310: 35-45.

24. Hannenhalli S, Kaestner KH. The evolution of Fox genes and their role in development and disease. Nat Rev Genet. 2009; 10: 233-240.

25. Zarbalis K, Siegenthaler JA, Choe Y, et al. Cortical dysplasia and skull defects in mice with a Foxc1 allele reveal the role of meningeal differentiation in regulating cortical development. Proc Natl Acad Sci U S A. 2007; 104: 14002-14007.

26. Aldinger KA, Lehmann OJ, Hudgins L, et al. FOXC1 is required for normal cerebellar development and is a major contributor to chromosome 6p25.3 Dandy-Walker malformation. Nat Genet. 2009; 41: 1037-1042.

27. Wang J, Ray PS, Sim MS, et al. FOXC1 regulates the functions of human basal-like breast cancer cells by activating NF-kappaB signaling. Oncogene. 2012.

28. Sizemore ST, Keri RA. The Forkhead Box Transcription Factor FOXC1 Promotes Breast Cancer Invasion by Inducing Matrix Metalloprotease 7 (MMP7) Expression. J Biol Chem. 2012; 287: 24631-24640.

29. Yilmaz M, Christofori G. EMT, the cytoskeleton, and cancer cell invasion. Cancer Metastasis Rev. 2009; 28: 15-33.

30. Sommer P, Napier HR, Hogan BL, et al. Identification of Tgf beta1i4 as a downstream target of Foxc1. Dev Growth Differ. 2006; 48: 297-308.

31. Hayashi H, Kume T. Forkhead transcription factors regulate expression of the chemokine receptor CXCR4 in endothelial cells and CXCL12-induced cell migration. Biochem Biophys Res Commun. 2008; 367: 584-589.

32. Qi J, Chen N, Wang J, et al. Transendothelial migration of melanoma cells involves N-cadherin-mediated adhesion and activation of the beta-catenin signaling pathway. Mol Biol Cell. 2005; 16: 4386-4397. 